\title{
Breath figures of two immiscible substances on a repellent surface
}

\author{
J. Guadarrama-Cetina and W. González-Viñas \\ Department of Physics and Applied Mathematics, University of Navarra, Navarra, Spain \\ (Received 25 September 2012; revised manuscript received 6 March 2013; published 20 May 2013)
}

\begin{abstract}
The understanding of the competition between different substances while condensing on a cold surface is of high interest in situations in which it is desirable to control their condensation rates and the formed morphologies. We do the experiments for mixtures of water and hexamethyldisiloxane vapors at several concentrations. The dropwise condensation of the vapors forms breath figures on a substrate that is repellant to both substances. We report the average radius of the drops for each specie as a function of time. Also, we pay attention to the evolution of the corresponding morphologies and the appearance of hybrid clusters.
\end{abstract}

DOI: 10.1103/PhysRevE.87.054401

PACS number(s): 68.03.Fg, 81.10.Bk, 87.15.nr, 82.70.Kj

\section{INTRODUCTION}

Water vapor condensation is a daily occurrence in nature, and the condensation of other vapors on surfaces is common in several industry sectors. In many situations, more than one kind of vapor condenses simultaneously. This leads to relevant phenomena. For example, the condensation of two different vapors may facilitate the catalysis of a chemical reaction $[1,2]$. Also, it is important to understand the competition between immiscible condensing vapors in petrochemical distillation [3] steam systems and solvent drying, as well as in humidity control systems [4] and in atmospheric condensers and heat exchangers. The formation of two-dimensional (2D) emulsions or patterns [5] could be of applied interest, too.

When two immiscible vapors condense at the same time, a complex dynamics appears in the formation of clusters which in turn perturb the growth of individual droplets. Thus, from a fundamental point of view, the behavior of the breath figure is affected by several mechanisms which have to be considered for the many different conditions encountered in possible applications.

In general, the dropwise heterogeneous condensation of vapors on cold surfaces [commonly known as breath figures (BFs)] [6-9] has three stages [10-12]: (i) the nucleation of drops, which is a thermally activated process, (ii) the growth of droplets by adding molecules to the already stable nuclei (this mechanism is related to the difference of partial pressure of the vapor in the atmosphere and the vapor pressure at the surface temperature), and (iii) a coalescence regime which is selfsimilar. The classical one-component BF has been studied for a long time both in theory and experiments. Fritter and Beysens [13] had obtained scaling laws to describe the intermediate stage and the coalescence stage. Family and Meakin $[10,12,14]$ and Briscoe et al. [15] proved the effect of heterogeneity and geometry during nucleation.

In other specialized literature, there are studies of water vapor condensing over immiscible substances to form patterns [16]. Other studies on metallurgy [17] report how Ga and $\mathrm{Pb}$ condense and distribute on a surface, which shows how relevant this study is to techniques such as physical vapor deposition. In this Brief Report, we present experimental results about the intermediate and coalescence stages. In particular, we study how the competition of gases at different concentrations affects the pattern formation. From the point of view of the already condensed substances and their occupation factors, we explore what experimental conditions effect on the observed pattern and what is the contribution of the two populations on the cluster formation.

\section{EXPERIMENTAL SETUP}

The condensation experiments were performed on common cover slip glasses coated with $3 \mathrm{M}$ ECG- $1700^{\circledR}$. This coating leaves on the glass a smooth hydro- and HMDSO-phobic surface that allows dropwise condensation of both substances. We evaporate hexamethyldisiloxane (HMDSO) and ultrapure water at room temperature $\left(T_{R} \approx 22^{\circ} \mathrm{C}\right)$ by bubbling air inside separated flasks to get saturated vapors, which are then directed to our colder substrate at $5^{\circ} \mathrm{C}$. We choose HMDSO and ultrapure water because they are immiscible and easy to evaporate. Also, both dew points (with respect to the room temperature) are comparable. The dew point temperatures are measured by flowing the saturated vapor at room temperature onto our surface at a decreasingly lower temperature until we observe condensation. HMDSO dew temperature is approximately $10^{\circ} \mathrm{C}$ under $T_{R}$. The dew temperature for ultrapure water is determined to be $1.5^{\circ} \mathrm{C}$ under $T_{R}$. The contact angle of HMDSO in common surfaces is very low, no more than $13^{\circ}$. However, on our coating, the advancing and receding contact angles are $\theta_{A}=29^{\circ}$ and $\theta_{R}=26^{\circ}$, respectively. The corresponding contact angles for ultrapure water are $\theta_{A}=108^{\circ}$ and $\theta_{R}=82^{\circ}$. These conditions allow water and HMDSO to condense dropwise simultaneously to study their competition. The substrate is put in the middle of the condensation chamber. It is a Delrin ${ }^{\circledR}$ cylindrical cavity where in the bottom part there is a copper plate in contact with a Peltier element which is, in turn, in contact with another small copper cylinder where recirculating water dissipates the heat. To avoid preferential condensation of HMDSO out of the substrate, the walls and the outer copper plate are also coated with 3M ECG-1700. All the observations have been done with an inline illumination microscope and recorded with a CMOS camera. The obtained images are analyzed through homemade routines in FORTRAN and OCTAVE to extract the average radius $\langle r\rangle$ of each component drop and the occupation factors or surface coverage $\varepsilon^{2}$, which is the ratio between the surface area occupied by the corresponding type of drop and the total observable area. 

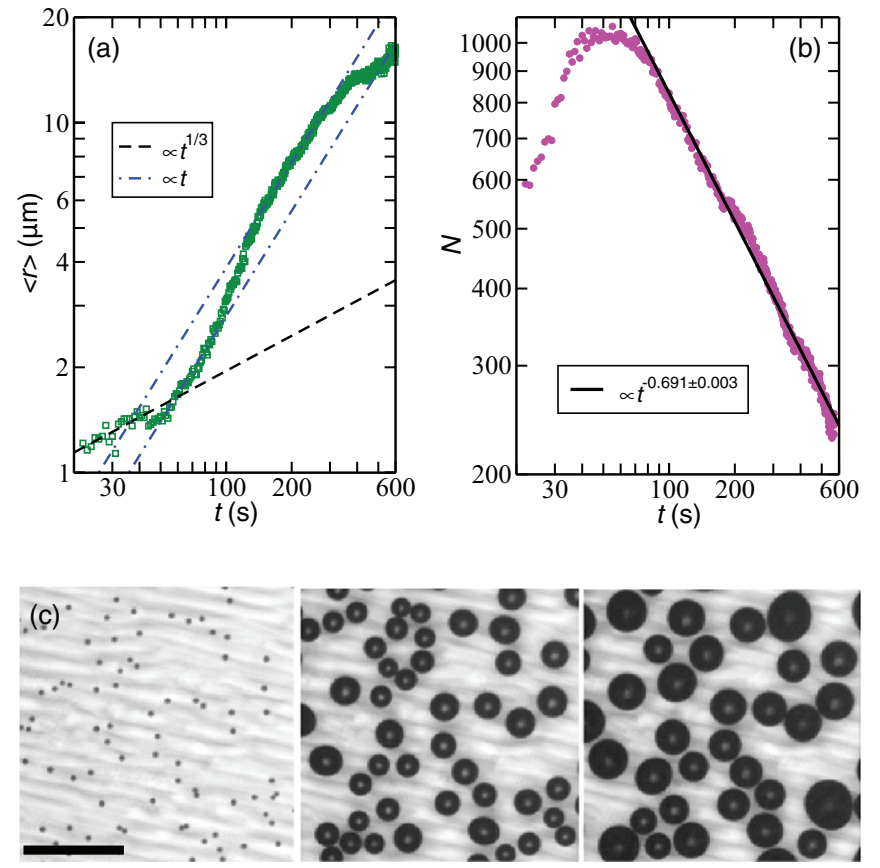

FIG. 1. (Color online) Classical water BF on our substrate. (a) Average radius (squares) and power laws $t^{1 / 3}$ (dashed line) and $t$ (dashed-dotted lines) for comparison. (b) Number of drops (circles) with a fit to a power law (solid line), and (c) three snapshots of the breath figure. Scale bar is $100 \mu \mathrm{m}$.

\section{RESULTS AND DISCUSSION}

As the coating leaves a smooth grooved surface, first we checked that classical condensation is produced on it. For that we flowed $(250 \mathrm{~mL} / \mathrm{min})$ saturated water vapor filtered air to the condensation chamber. In Fig. 1(a), we show that the growth laws reproduce the expected power laws with exponents $1 / 3$ (in the free growth regime) and 1 (in the self-similar coalescence regime). In the latter, we observe an anomaly during a short period of time, which coincides when the size of the droplets is of the order of the grooving length scale. It can also be observed that the number of drops in the self-similar regimes scales as $t^{-0.691 \pm 0.003}$. The occupation factor in the coalescence stage was measured as $\varepsilon^{2} \approx 0.63$. Thus, we conclude that the classical BF [18] occurs and that the effect of the specific surface we use is minimal.

Before commencing the experiments with the two immiscible substances together, we performed an experiment with the HDMSO alone. The condensation of HMDSO evolves in a different manner than water condensation. In our experimental conditions, we observed from the very beginning coalescence events. Also, we could see, at relatively small times, the appearance of new generations of drops coming from new nucleating sites. Consistently, we obtained a linear law for the growth of HMDSO [Fig. 2(a)]. Here, there is a departure from the expected behavior when there is no statistically dominant family or generation. This kind of behavior is also mirrored in the number of droplets, which follows a power law with exponent $-2.02 \pm 0.04$. Here the occupation factor in the coalescence stage is $\varepsilon^{2} \approx 0.8$, which agrees with a smaller contact angle in relation with water. Consequently, in our
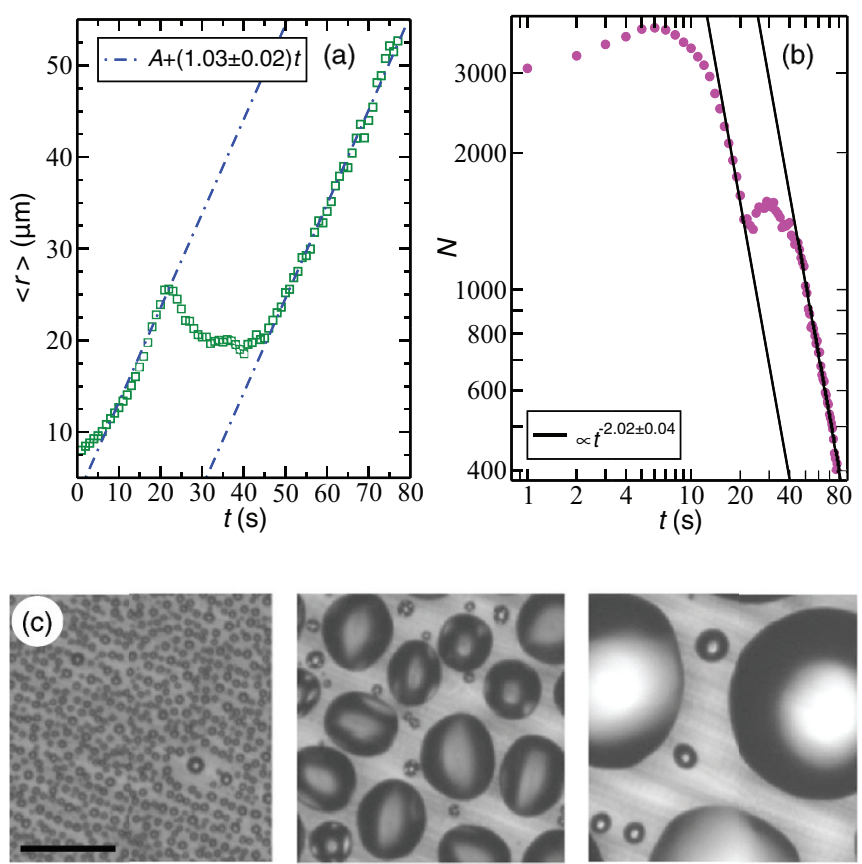

FIG. 2. (Color online) HMDSO BF on our substrate. (a) Average radius (squares) and a linear fit (dashed line) to the rightmost data and a translation of the fit to the left for comparison. (b) Number of drops (circles) with a power-law fit to the rightmost data (solid line) and a scale of the fit to the left for comparison, and (c) three snapshots of the breath figure. Scale bar is $100 \mu \mathrm{m}$.

conditions we cannot observe the classical behavior of the free-growth regime of the droplets.

We were first interested in how HDMSO does condense on a previous water BF. For that, we first condensed water vapor on our substrate by flowing water-saturated filtered air at room temperature to the colder substrate $\left(5^{\circ} \mathrm{C}\right)$ until we approximately reached the desired conditions (occupation factor and size of the droplets). Then the flow was stopped and we waited until we obtained a (nearly) stationary BF. Then, HMDSO saturated in dry filtered air at $T_{R}$ was flowed over the same substrate. In Fig. 3, it is possible to observe a typical condensation pattern where there has not been coalescence between

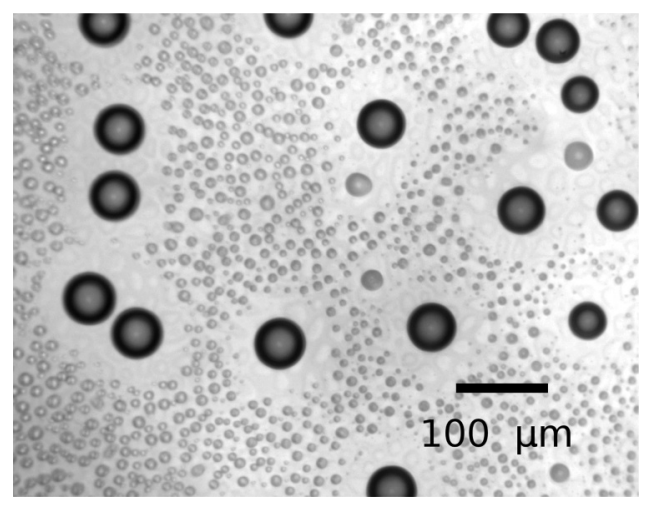

FIG. 3. First stage after HMDSO saturated vapor is flowed over a water breath figure (darker droplets). The lighter smaller droplets correspond to HMDSO. Concentric regions to the drops, where condensation is inhibited, can be observed. 


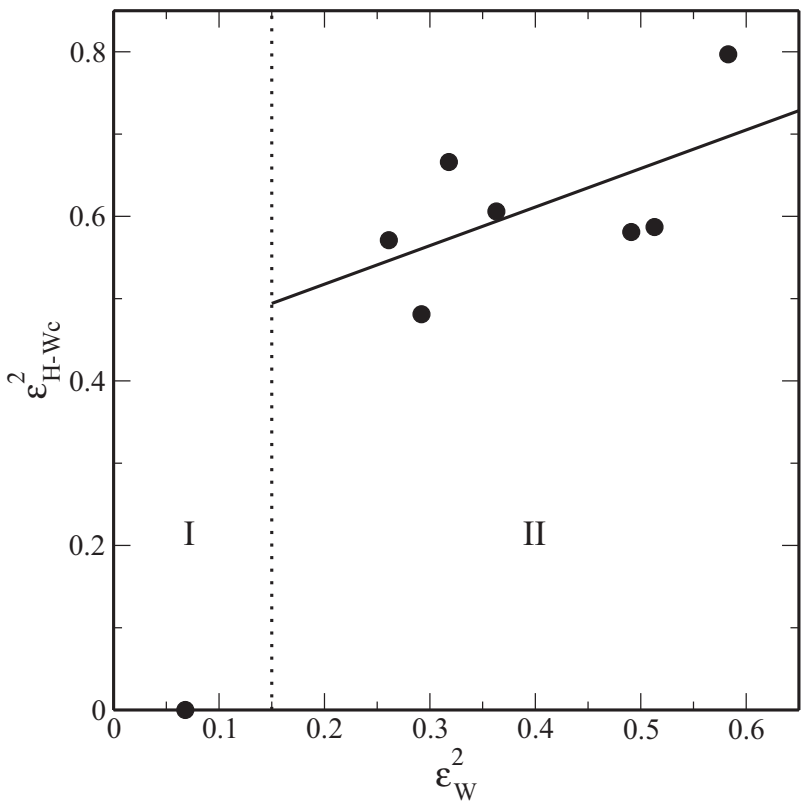

FIG. 4. Occupation factor for $\mathrm{H}-\mathrm{Wc} \varepsilon_{\mathrm{H}-\mathrm{Wc}}$ as the initial occupation factor for the water $\mathrm{BF}$ changes. The dotted line is a guide for the eye and it classifies the morphologies obtained. I stands for HMDSO ponds with water droplets immersed in them, and II stands for $\mathrm{H}-\mathrm{Wc}$ regions. The solid line is a linear fit to the data excluding region I.

different component drops yet. Surrounding the water drops (dark large droplets) and the biggest HMDSO droplets, there is a depletion zone where no condensation is produced [18]. The depletion zone reduces as time increases. As a consequence, HMDSO can condense everywhere. This leads to increased coalescence and to the ability to condense new generations of droplets.

After some time, water and HMDSO droplets are brought together by surface tension forces. As both substances are immiscible, they form clusters which hereafter will be called H-Wc [for an example at their beginning stage, see Fig. 5(a), center]. The pattern morphology obtained at long times depends on the initial water droplet occupation factor $\varepsilon_{\mathrm{W}}^{2}$. In Fig. 4, we observe that for very small water occupation factors, HMDSO vapor will condense into ponds and will not form H-Wc. At bigger $\varepsilon_{\mathrm{W}}^{2}, \mathrm{H}-\mathrm{Wc}$ will appear and then $\varepsilon_{\mathrm{H}-\mathrm{Wc}}$ increases monotonically with $\varepsilon_{\mathrm{W}}^{2}$. Hence, the $\mathrm{H}-\mathrm{Wc}$ formation varies largely due to the interaction of the two substances on the liquid phase.

When we tried the opposite experiment condensing first HMDSO, we only observed the production of large HMDSO ponds with water droplets immersed in them. After this experience, we bubble-filtered dry air through ultrapure water and HMDSO at constant flux and at room temperature $T_{R}$. The vapors were directed to a separate vessel where they were mixed and then were directed to the coated surface at $5{ }^{\circ} \mathrm{C}$ inside the condensation chamber. In this case, we observed that, under our range of experimental conditions, water condenses first (Fig. 5, left). After some time, HMDSO condenses at a very fast rate. Then, HMDSO drops mostly grow by coalescence and are able to create new generations of droplets (Fig. 5, center). For larger values of time, we could also observe how HMDSO can condense on water droplets
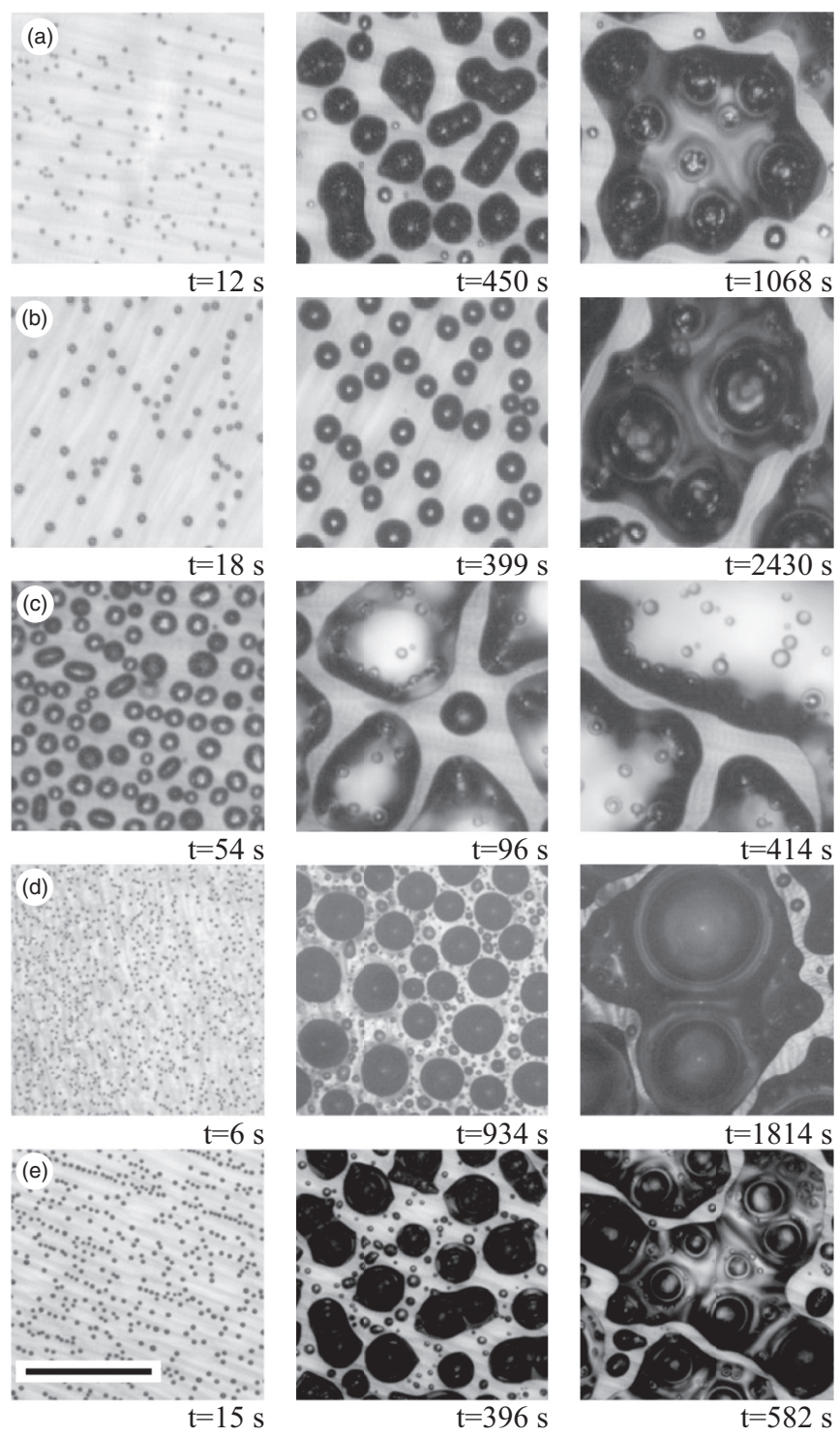

FIG. 5. BF evolution snapshots for HMDSO and water at different fluxes of water-HMDSO (mL/min) for increasing times. (a) 250-230, (b) 250-240, (c) 250-250, (d) 240-250, and (e) 230-250. Scale bar is $152 \mu \mathrm{m}$.

and enter into them. Water droplets mostly grow without coalescence, except when the condensation of HMDSO induces large water droplets to coalesce with other water droplets. This induction by the liquid HMDSO allows the water droplets to increase their size and to reduce their interspace, driving the pattern of two separate substances toward a pattern of water droplets immersed in the HMDSO [Fig. 5(c), right] or toward H-Wc [Figs. 5(a), 5(b), 5(d), and 5(e), right].

In Fig. 5, we show the representative evolution of the BF at several flux ratios. At long times (right) we can see $\mathrm{H}-\mathrm{Wc}$ and water droplets in HMDSO ponds.

We measured $\langle r\rangle$ for water and HMDSO. In the case of the coalescence stage, we measured $\langle r\rangle$ for HMDSO because its droplets can always be found in water-depleted regions. Although in this regime we do not observe isolated water droplets, we obtained $\langle r\rangle$ for H-Wc. In Fig. 6, the values of $\langle r\rangle$ are compared with the power laws with exponents $1 / 3$ 


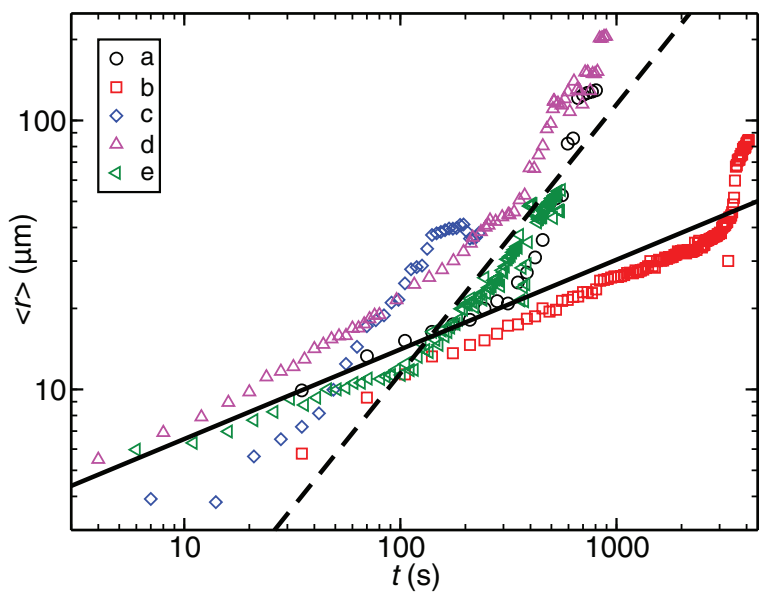

FIG. 6. (Color online) The average radius $\langle r\rangle$ for the water droplets in the BF presents two stages: the small values represent the intermediate stage close to a power law with exponent $1 / 3$ (solid line). The rest of the values represent the mean size of the H-Wc (see text). The dashed line is a guide for the eye representing a power law with exponent 1 . The coding $(\mathrm{a}-\mathrm{e})$ for the fluxes follows that of Fig. 5.

and 1 . The already formed water droplets at the intermediate stage grow by the absorption of the molecules on the surface near the contact line. At this stage, the droplets increase their size by this mechanism [19]. This impedes the nucleation of the HMDSO drops at the beginning. Once the water droplets reach a critical size, or the concentration of molecules is near zero, the activity of absorption of those molecules reaches a minimum. As a consequence, the HMDSO molecules finally condense on the surface. In Fig. 7, the values of $\langle r\rangle$ are shown. They cannot be properly fitted by power laws of exponents $1 / 3$ or 1 (power laws with these exponents are not shown). Also they cannot be represented by linear fits with different origins, as occurs in Fig. 2. Together with the data, a power-law fit is represented with exponent $1.8 \pm 0.1$. This increased exponent could be due to the fact that newly born generations of HMDSO droplets occur randomly over the substrate and they become increasingly absorbed by $\mathrm{H}-\mathrm{Wc}$ or by water droplets. Thus the drops which survive are bigger as time evolves, leading to an effective faster growth. Also, another possible explanation could be that the effective dimension of the substrate is bigger than 2 [19] when clusters are on the surface, and this may lead to an increased growth of HMDSO drops in the coalescence stage.

\section{CONCLUDING REMARKS}

We described the interaction of two immiscible vapors by the analysis of their occupation factor $\left(\varepsilon^{2}\right)$ and the mean radius

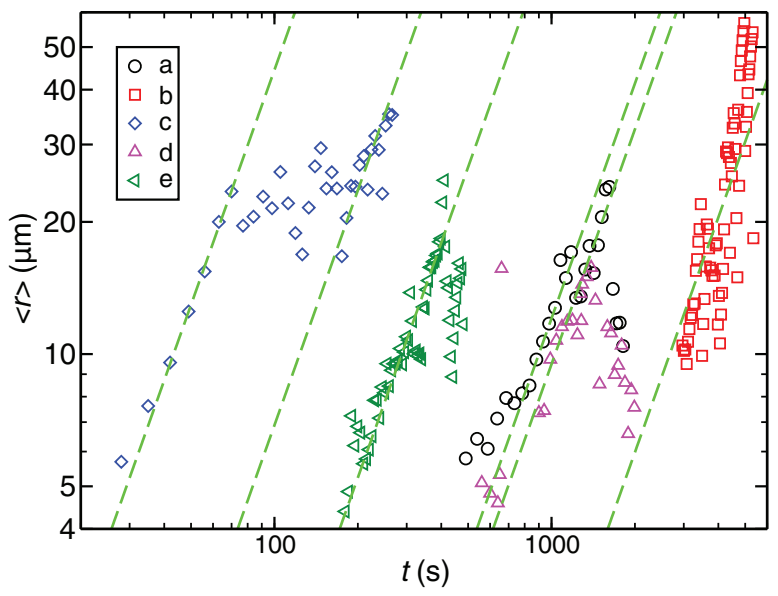

FIG. 7. (Color online) The average radius $\langle r\rangle$ for the HMDSO droplets in the BF and a power-law fit to the leftmost data (dashed line) and scales of the fit to the right for comparison. The coding (a-e) for the fluxes follows that of Fig. 5 .

size $\langle r\rangle$ of the droplets for each substance. In a first stage, we showed that the coating used is suitable to reproduce classical water BF. However, the HMDSO BF occurred in our experimental conditions without the (free) growth regime. This could be related either to the fact that after some small time the depletion zones disappear from the surroundings of the droplets, or to the fact that the dynamics is too fast to be observed. It would be interesting if further experiments could deal with this issue at lower fluxes (and consequently slower dynamics) or with better time-resolved observation. Nevertheless, when the two vapors are mixed, we observe anomalous growth rates for the HMDSO, although water droplets follow very slightly modified growth. This deviation could be related to the appearance of $\mathrm{H}-\mathrm{Wc}$. In the experiments of condensation of HMDSO on a previously formed water BF, we observed that clusters of HMDSO and water $(\mathrm{H}-\mathrm{Wc})$ only are produced when the $\mathrm{BF}$ of water has large values of $\varepsilon^{2}$. Concerning the flux rates and the mixed vapors, we conclude that these are secondary factors to the phenomena reported in this Brief Report, although it is clear from Figs. 6 and 7 that they have some not yet understood effect, which require further research into this kind of system.

\section{ACKNOWLEDGMENTS}

We thank an anonymous reviewer for his or her remarks, R. Narhe for suggesting the experiment, and D. Beysens and R. Narhe for fruitful discussions. This work was partly supported by the Spanish MEC (Grant No. FIS2011-24642) and by Departamento de Educación (Gobierno de Navarra). J.G.C. acknowledges financial support from the "Asociación de Amigos de la Universidad de Navarra."
[1] J. Ji, Y. Zhao, L. Guo, B. Liu, C. Ji, and P. Yang, Lab Chip 12, 1373 (2012).

[2] R. G. Sardesai and D. R. Webb, Chem. Eng. Sci. 37, 529 (1982).
[3] O. Tutkun, Chem. Eng. Proc. 32, 263 (1993).

[4] V. D. Rao, V. M. Krishna, K. Sharma, and P. M. Rao, Int. J. Heat Mass Transf. 51, 6090 (2008). 
[5] P. Borckmans, G. Dewel, A. D. Wit, E. Dulos, J. Boissonade, F. Gauffre, and P. D. Kepper, Int. J. Bifurcation Chaos 12, 2307 (2002).

[6] J. Aitken, Proc. R. Soc. London 51, 408 (1892).

[7] L. Rayleigh, Nature (London) 86, 416 (1911).

[8] T. Baker, Philos. Mag. Ser. 6 44, 752 (1922).

[9] B. Lewis and J. Anderson, Nucleation and Growth of Thin Films (Academic, New York, 1978).

[10] P. Meakin and F. Family, J. Phys. A 22, L225 (1989).

[11] A. Steyer, P. Guenoun, and D. Beysens, Phys. Rev. Lett. 68, 64 (1992).
[12] P. Meakin, Rep. Prog. Phys. 55, 157 (1992).

[13] D. Fritter, C. M. Knobler, and D. A. Beysens, Phys. Rev. A 43, 2858 (1991).

[14] F. Family and P. Meakin, Phys. Rev. A 40, 3836 (1989).

[15] B. J. Briscoe and K. P. Galvin, J. Phys. D 23, 1265 (1990).

[16] U. Bunz, Adv. Mater. 18, 973 (2006).

[17] R. Kofman, M. Allione, F. Celestin, Z. Barkay, and Y. Lereah, Eur. Phys. J. D 50, 279 (2008).

[18] D. Beysens and C. M. Knobler, Phys. Rev. Lett. 57, 1433 (1986).

[19] D. Beysens, C. R. Phys. 7, 1082 (2006). 